\title{
Class A1 scavenger receptors mediated macrophages in impaired intestinal barrier of inflammatory bowel disease
}

\author{
Chenxi Xie ${ }^{1 \#}$, Yanyun Fan ${ }^{1 \#}$, Yinshi Huang ${ }^{2 \#}$, Shuangting $\mathrm{Wu}^{1}$, Haimei $\mathrm{Xu}^{1}$, Lupeng Liu ${ }^{1}$, Yiqun $\mathrm{Hu}^{1}$, \\ Qingwen Huang ${ }^{1}$, Huaxiu Shi ${ }^{1}$, Lin Wang ${ }^{1}$, Hongzhi Xu ${ }^{1}$, Jingling Su ${ }^{1}$, Jianlin Ren ${ }^{1}$ \\ ${ }^{1}$ Department of Gastroenterology, Affiliated Zhongshan Hospital of Xiamen University, Xiamen 361004, China; ${ }^{2}$ Department of Gastroenterology, \\ Shanghai General Hospital, Shanghai Jiao Tong University, School of Medicine, Shanghai 201620, China \\ Contributions: (I) Conception and design: C Xie, Y Fan, Y Huang, J Su, J Ren; (II) Administrative support: J Su; (III) Provision of study materials \\ or patients: C Xie, Y Fan, Y Huang, S Wu, H Xu, L Liu, Y Hu; (IV) Collection and assembly of data: Q Huang, H Shi, L Wang, H Xu; (V) Data \\ analysis and interpretation: C Xie, Y Fan, Y Huang; (VI) Manuscript writing: All authors; (VII) Final approval of manuscript: All authors. \\ \#These authors contributed equally to this work. \\ Correspondence to: Jingling Su; Jianlin Ren. Department of Gastroenterology, Affiliated Zhongshan Hospital of Xiamen University, Xiamen 361004, \\ China. Email: sujingling@163.com; renjianl@xmu.edu.cn.
}

\begin{abstract}
Background: This study was to investigate the cytokines and phenotype of macrophages pre-treated with class A1 scavenger receptor (SR-A1) antibody in vitro and the influence on apoptotic pathway of colonic epithelial cells, and to explore the role of SR-A1 mediated macrophages in impaired intestinal barrier of inflammatory bowel diseases (IBDs).

Methods: Mouse macrophage RAW264.7 was pre-treated with SR-A1 antibody in the presence of lipopolysaccharide (LPS). Transwell system was employed for co-culture of RAW264.7 and Caco-2 in the presence of LPS and IFN- $\gamma$, with or without SR-A1 antibody pre-treatment. The percentage of $\mathrm{F} 4 / 80^{+} \mathrm{CD} 11 \mathrm{c}^{+}$macrophages, apoptosis rate of Caco-2 cells, and expression of apoptosis and tight junction proteins in Caco-2 cells was determined.

Results: Pre-treatment with SR-A1 antibody up-regulated IL-10 expression in RAW264.7, whereas downregulated the expression of TNF and iNOS. Immunofluorescence staining indicated the upregulation of NF- $\kappa$ B p-p56 after LPS stimulation was significantly inhibited in the presence of SR-A1 antibody. The increase in p-JNK expression was inhibited by SR-A1 antibody. Transwell assay showed the percentage of $\mathrm{F} 4 / 80^{+} \mathrm{CD} 11 \mathrm{c}^{+}$macrophages and apoptotic Caco-2 cells increased after treatment with LPS and IFN- $\gamma$, which could be reversed in the presence of SR-A1 antibody. The induction of cleaved caspase- 3 and claudin-1 in Caco-2 cells was also suppressed when SR-A1 antibody pre-treatment.

Conclusions: Pre-treatment with SR-A1 antibody can inhibit inflammatory response in LPS-induced macrophages in a NF- $\kappa \mathrm{B}$ dependent manner. Pre-treatment with SR-A1 antibody also inhibits M1 phenotype expression of macrophages, and attenuates the pro-apoptotic effect on colonic epithelial cells and disruption of intestinal barrier integrity induced by macrophages.
\end{abstract}

Keywords: Macrophages; inflammatory bowel disease (IBD); NF-кB

Submitted May 08, 2019. Accepted for publication Aug 14, 2019.

doi: 10.21037/atm.2019.12.107

View this article at: http://dx.doi.org/10.21037/atm.2019.12.107 


\section{Introduction}

Inflammatory bowel disease (IBD), comprising Crohn's disease (CD) and ulcerative colitis (UC) are idiopathic inflammatory disorders of unknown cause and genetic, immunologic, and environmental factors are found to be involved in the pathogenesis of these diseases $(1,2)$. As a subset of innate immune cells, macrophages play a critical role in maintaining mucosal homeostasis and defensing pathogens invasion $(3,4)$. Macrophages can be divided into two subtypes: the classically activated macrophages (M1 macrophage) which can produce a large amount of reactive oxygen species (ROS) and cytokines and mediate inflammatory response, and the alternatively activated macrophage (M2 macrophages) which may promote tissue repair and remodeling (5). The imbalance of M1/ M2 paradigm can lead to the development of chronic inflammatory disorders such as IBD, characterized by the increased production of IL- $1 \beta$, TNF, IL- 6 and iNOS, and the decreased production of IL-10 in mucosal macrophages, which can impair the integrity of intestinal mucosal barrier. Currently, treatments for IBD are non-specific, and macrophages may serve as an effective target in the treatment of IBD.

Class A1 scavenger receptor (SR-A1) is a pattern recognition receptor (PRR) primarily expressed in macrophages (6). SR-A1 has primarily been considered as an important factor in anti-atherosclerosis because of it originally internalizes chemically modified lowdensity lipoprotein (7). There is evidence showing SRA1 is implicated in the process of inflammatory response in case of tissue injury (8). Recent studies have shown that malondialdehyde-acetaldehyde (MAA)-induced lung inflammation is attenuated in the absence of SR-A1 (9). Maleylated bovine serum albumin (M-BSA), as a SR-A1 competitive inhibitor, has been reported to ameliorate lipopolysaccharide (LPS)-induced sepsis and dextran sulfate sodium (DSS)-induced colitis in animal models, which indicate that SR-A1 plays a crucial role in the innate immune response in case of intestinal inflammation (10).

The present study aimed to investigate the change of inflammatory cytokines, transcription factor, proliferative protein and phenotype of mouse macrophages induced by LPS. In addition, mouse macrophages were cocultured with colonic epithelial cells, and the influence of macrophages on the intestinal mucosal barrier was explored via detecting macrophage polarization, apoptosis pathway and the expression of tight junction proteins.

\section{Methods}

\section{Cell culture}

Mouse macrophage cell line RAW264.7 cells and human colonic epithelial cell line Caco-2 cells were obtained from the ATCC (Rockville, USA), and maintained in DMEM medium (Gibco, USA) supplemented with 10\% fetal bovine serum (FBS; Life Technologies, USA) in a humidified atmosphere at $37{ }^{\circ} \mathrm{C}$ with $5 \% \mathrm{CO}_{2}$. RAW264.7 cells were pre-treated with $0.6 \mu \mathrm{g} / \mathrm{mL}$ SR-A1 antibody for $6 \mathrm{~h}(\mathrm{R} \& \mathrm{D}$, USA) in vitro, and then treated for $24 \mathrm{~h}$ with $1 \mu \mathrm{g} / \mathrm{mL}$ LPS (Sigma, USA). Cells without SR-A1 antibody treatment served as a control group, and cells without SR-A1 antibody and LPS treatments served as a negative group.

\section{Antibodies}

Rabbit anti-STAT6 monoclonal antibody (mAb), rabbit anti cleaved caspase- $3 \mathrm{mAb}$, Rabbit anti-ERK1/2 mAb, rabbit anti-p-ERK1/2 mAb, rabbit anti-JNK, rabbit anti-pJNK, rabbit anti-p38 and rabbit anti-p-p38 were purchased from Cell Signaling Technology Ltd. (USA). Rabbit antioccludin were purchased from Abcam Ltd. (USA). FITCconjugated anti-mouse F4/80, and APC-conjugated antimouse CD11c were purchased from Termo Fisher, USA. Zonulin-1 and claudin-1 were purchased from Santa Cruz Ltd. (USA). Anti-GAPDH, goat anti-Mouse, and goat antirabbit were purchased from Lianke Ltd. (USA).

\section{Quantitative real-time polymerase chain reaction (qRT-PCR)}

Total RNA was extracted from RAW264.7 cells and the cDNA were synthesized with SYBR PrimeScript RT reagent kit (TaKaRa Dalian, China) according to the manufacturer's instructions. qRT-PCR was performed with the ABI prism $7900 \mathrm{HT}$ thermal cycler (Applied Biosystems, Foster City, CA, USA) using SYBR green methodology. PCR was performed in triplicate wells using the following conditions: $95^{\circ} \mathrm{C}$ for $1 \mathrm{~min}$, followed by 40 cycles of $95^{\circ} \mathrm{C}$ for $15 \mathrm{sec}$ and $60^{\circ} \mathrm{C}$ for $30 \mathrm{sec}$. The relative mRNA expressions were calculated by normalizing to $\beta$-actin mRNA expression.

The primers for PCR were as follows: IL-10: forward 5'-CTTACTGACTGGCATGAGGATCA-3', reverse 5'-GCAGCTCTAGGAGCATGTGG-3'; TNF: forward 5' -ATGAGCACAGAAAGCATGATC-3', reverse 5'-CTCATCCCTTTGGGGACCGAT-3'; iNOS : 
forward 5'-GTTCTCAGCCCAACAATACAAGA-3', reverse 5'-GTGGACGGGTCGATGTCAC-3'; $\beta$-actin: forward 5'-AGAGGGAAATCGTGCGTGAC-3', reverse 5'-AGGAAGAGGATGCGGCAGT-3'.

\section{Immunofluorescence staining}

RAW264.7 cells were fixed in 4\% paraformaldehyde and incubated with primary antibody p-p65 (Santa Cruz, USA) at $4{ }^{\circ} \mathrm{C}$ overnight. After addition of fluorescent secondary antibody (Invitrogen, Carlsbad, USA), incubation was done for $30 \mathrm{~min}$. 2-(4-amidinophenyl)-6-indolecarbamidine dihydrochloride (DAPI; $2 \mathrm{mg} / \mathrm{mL}$; Beyotime, China) was used to stain the cell nuclei. Immunofluorescence was observed under a fluorescence microscope (Olympus BX43, Japan).

\section{Western blotting}

Total protein was extracted from RAW264.7 cells and Caco-2 cells by using RIPA buffer with a cocktail of protease and phosphatase inhibitors. After quantification of protein concentration using BCA assay by microplate spectrophotometry (Thermo, Massachusetts, USA), proteins were separated by sodium dodecyl sulfate-polyacrylamide gel electrophoresis (SDS-PAGE) and then transferred onto nitrocellulose membranes (Millipore, Massachusetts, USA). Membranes were treated with primary antibodies at $4{ }^{\circ} \mathrm{C}$ overnight. After incubation with secondary antibody, immunoblot detection was performed with the Odyssey Imaging System (Li-COR Biosciences, Nebraska, USA).

\section{Transwell chamber assay}

The Transwell system was employed for the co-culture of RAW264.7 cells and Caco-2 cells as previously reported (11). In brief, RAW264.7 cells $\left(8.5 \times 10^{5} /\right.$ well $)$ were added to 6-well plates, and Caco- 2 cells $\left(3.75 \times 10^{5} /\right.$ well $)$ were seeded as a cell monolayer on a polycarbonate membrane inserted in the Transwell system (Life Sciences, USA). RAW264.7 cells were pre-treated with SR-A1 antibody $(0.6 \mu \mathrm{g} / \mathrm{mL})$ for $6 \mathrm{~h}$, then treated with $1 \mu \mathrm{g} / \mathrm{mL}$ LPS and $2.5 \mathrm{ng} / \mathrm{mL} \mathrm{IFN-} \gamma$ for 24 h. Cells without SR-A1 antibody treatment, cells without SR-A1 antibody and cytokines treatment, and RAW264.7 cells alone served as control groups. RAW264.7 and Caco2 cells in the Transwell system were harvested for further examinations.

\section{Flow cytometry}

RAW264.7 cells were washed with cold PBS, re-suspended in $100 \mu \mathrm{L}$ of PBS and stained with FITC-conjugated antimouse F4/80 (Termo Fisher, USA) and APC-conjugated antimouse CD11c (Termo Fisher, USA) antibodies at $2 \mu \mathrm{g} / \mathrm{mL}$ for $30 \mathrm{~min}$ at $4{ }^{\circ} \mathrm{C}$. Finally, cells were fixed in $0.5 \mathrm{~mL}$ of $3 \%$ paraformaldehyde, and subjected to flow cytometry on the FACS Calibur instrument (BD Biosciences, CA, USA). Data were analyzed with CellQuest software.

\section{Statistical analysis}

All data are expressed as mean \pm standard error of the mean (SEM). Statistical analysis was performed using SPSS Statistics version 21.0 (SPSS, Chicago, IL, USA). Comparisons between two groups were performed using $t$-test or one-way analysis of variance for comparison of three or more groups as indicated. A value of $\mathrm{P}<0.05$ was considered statistically significant.

\section{Results}

SR-A1 antibody pre-treatment up-regulated antiinflammatory cytokines and down-regulated inflammatory cytokines secreted by RAW264.7 cells in a NF- $\kappa B$ patbway dependent manner

Macrophages are a type of primary innate immune cells in the inflammatory response, especially in the intestinal mucosa. Intestinal macrophages involve in regulating inflammation by producing inflammatory cytokines and chemokines, such as TNF and iNOS. Furthermore, macrophages can also play a protective role in inflammation is through producing IL-10 to repair mucosal tissues $(12,13)$. The expression of several inflammatory or antiinflammatory mediators was detected by RT-PCR. Compared with negative control, the mRNA expression of TNF and iNOS increased significantly, but that of IL-10 decreased markedly after LPS treatment in RAW264.7 cells $(\mathrm{P}<0.05)$. Pre-treatment with SR-A1 antibody dramatically up-regulated IL-10 expression in RAW264.7 cells, whereas down-regulated the expression of TNF and iNOS $(\mathrm{P}<0.05$ vs. LPS group) (Figure 1).

NF- $\kappa \mathrm{B}$ plays an important role and is responsible for the production of cytokines and chemokines in macrophageinduced inflammation (14). As shown in Figure 2, NF$\kappa \mathrm{B}$ p-p56 expression was mainly found in the cell nuclei, 


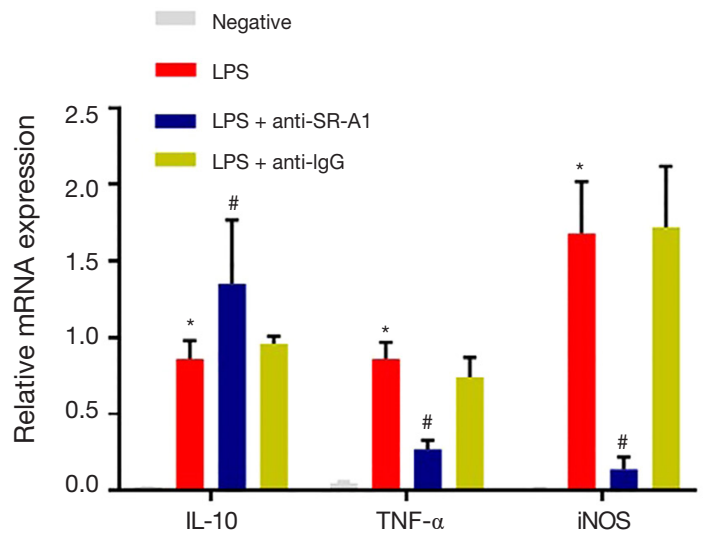

Figure 1 SR-A1 antibody pre-treatment up-regulates antiinflammatory cytokine (IL-10) and down-regulates inflammatory cytokines (TNF and iNOS) secreted by RAW264.7 cells. Expression of cytokines in RAW264.7 cells was detected by qPCR. Means were from three independent experiments. Data are presented as mean \pm SEM $\left({ }^{*}, \mathrm{P}<0.05\right.$ vs. negative group; ${ }^{\#}, \mathrm{P}<0.05$ $v s$. LPS group). SR-A1, A1 scavenger receptor; qPCR, quantitative polymerase chain reaction; SEM, standard error of the mean; LPS, lipopolysaccharide. and NF- $\mathrm{NB}$ p-p56 expression was upregulated after LPS stimulation $(\mathrm{P}<0.05)$, which however was significantly inhibited in the presence of SR-A1 antibody pre-treatment. These findings indicate pre-treatment with SR-A1 antibody may up-regulate anti-inflammatory cytokines and downregulate inflammatory cytokines secreted by RAW264.7 cells in a NF- $\kappa \mathrm{B}$ pathway dependent manner.

\section{Effects of SR-A1 antibody on the expression of proliferation related proteins in $R A W 264.7$ cells}

The expression of p38, ERK, JNK and corresponding phosphorylated proteins was detected by Western blotting. As shown in Figure 3, the expression of p-ERK1/2, JNK and p-JNK increased significantly after LPS stimulation $(\mathrm{P}<0.05)$. However, only the increased $\mathrm{p}-\mathrm{JNK}$ expression could be inhibited in the presence of SR-A1 antibody $(\mathrm{P}<0.05$ vs. LPS group). We also detected the expression of SR-A1 by Western blotting, which indicated that SR-A1 was present in RAW 264.7 cells.
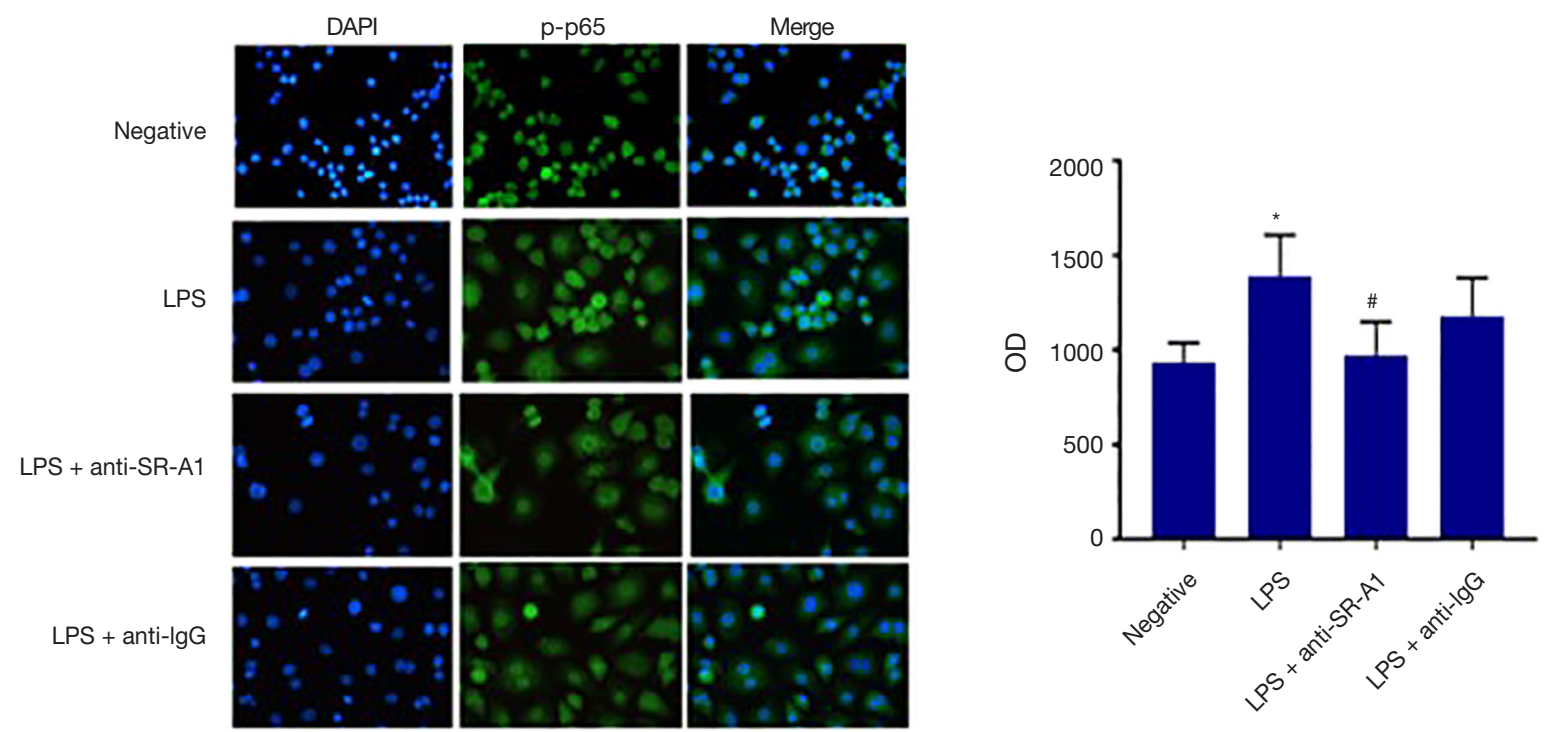

Figure 2 NF- $\kappa \mathrm{B}$ p-p65 expression in RAW264.7 cells with different treatments. NF- $\kappa \mathrm{B}$ p-p65 expression was determined by immunofluorescence staining. Nuclei were stained with DAPI, and green signal represented NF- $\kappa \mathrm{B}$ p-p65 $(\times 100)$. Data are presented as the mean \pm SEM (*, $\mathrm{P}<0.05$ vs. negative group; ${ }^{\prime}, \mathrm{P}<0.05$ vs. LPS group). SEM, standard error of the mean; LPS, lipopolysaccharide; SR-A1, A1 scavenger receptor; OD, optical density. 

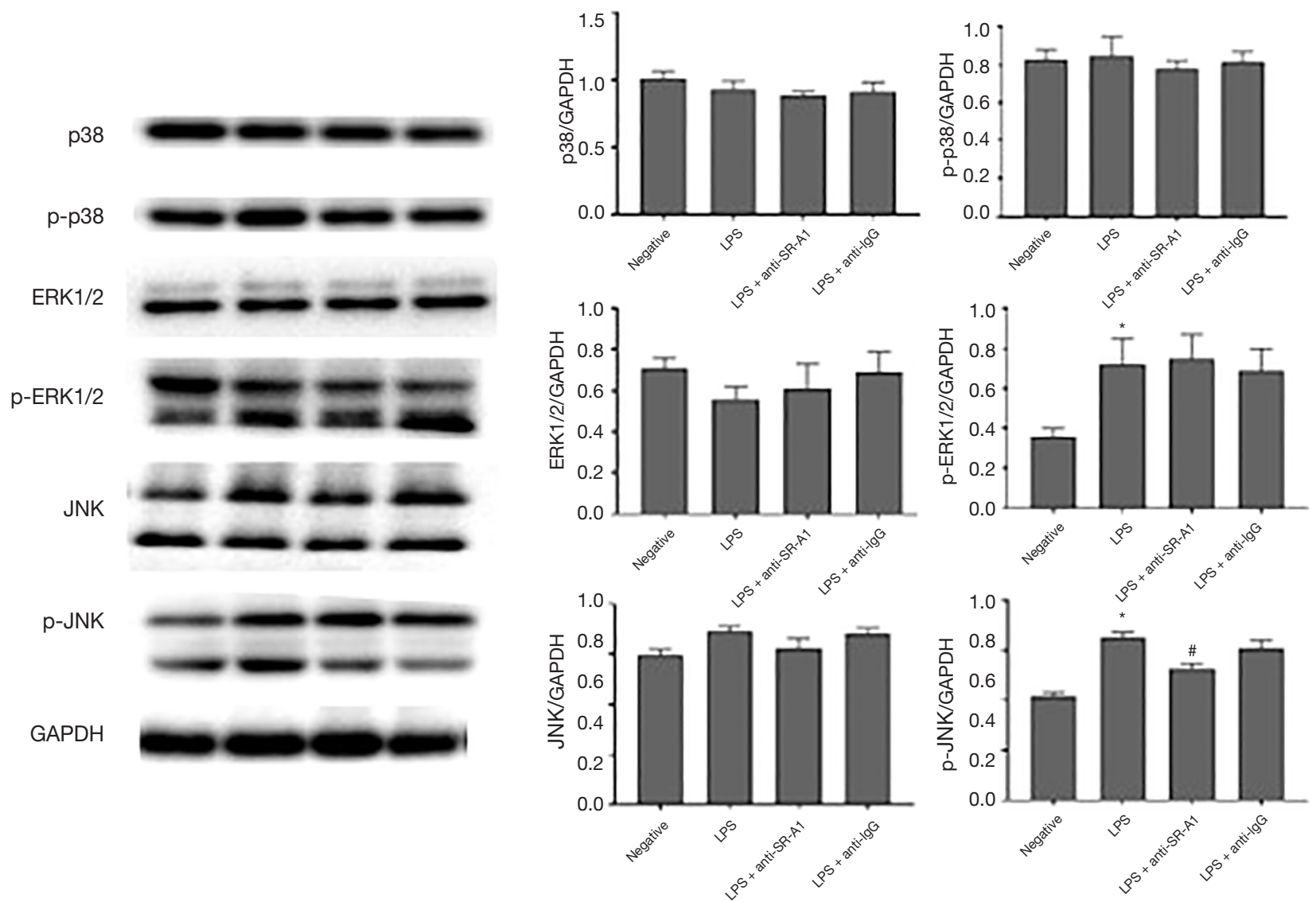

Figure 3 Expression of proliferation related protein in RAW264.7 cells with different treatments. Protein expression was detected by Western blotting. Data are presented as mean \pm SEM $\left({ }^{*}, \mathrm{P}<0.05\right.$ vs. negative group; ${ }^{*}, \mathrm{P}<0.05$ vs. LPS group). SEM, standard error of the mean; LPS, lipopolysaccharide; SR-A1, A1 scavenger receptor.

\section{Pre-treatment with SR-A1 antibody inbibited M1 phenotype of RAW264.7 cells independent of STAT6 patbway}

Macrophages can be classified as pro-inflammatory M1 macrophages and anti-inflammatory M2 macrophages, which have the ability to change phenotype and function according to surrounding environment (15). The STAT6 pathway has been found to play an important role in M1M2 polarization (16). To determine whether SR-A1 can contribute to the polarization of M1-M2 paradigm in the presence of LPS and IFN- $\gamma$, flow cytometry was done to evaluate the phenotype of RAW264.7 cells after pretreatment with SR-A1 antibody in the co-culture system. Compared with other control groups, $\mathrm{F} 4 / 80^{+} \mathrm{CD} 11 \mathrm{c}^{+}$ macrophages increased significantly after the treatment with LPS and IFN- $\gamma(\mathrm{P}<0.05)$, which could be reversed by pre-treatment with SR-A1 antibody. These suggest M1 polarization of macrophages is inhibited by SR-A1 antibody pre-treatment (Figure 4).

The protein expression of STAT6 in RAW264.7 cells was detected by western blotting. Results showed the protein expression of STAT6 slightly increased in the presence of LPS and IFN- $\gamma$ and slightly decreased in the presence of SR-A1 antibody pre-treatment ( $>0.05$ vs. LPS group) (Figure 5).

\section{SR-A1 antibody pre-treatment attenuated pro-apoptotic effect of RAW264.7 cells on Caco-2 cells in the co-culture system}

Previous studies indicated that macrophage can exert its pro-apoptosis function though SR-A1 receptor, finally inducing the expression of pro-apoptotic protein 

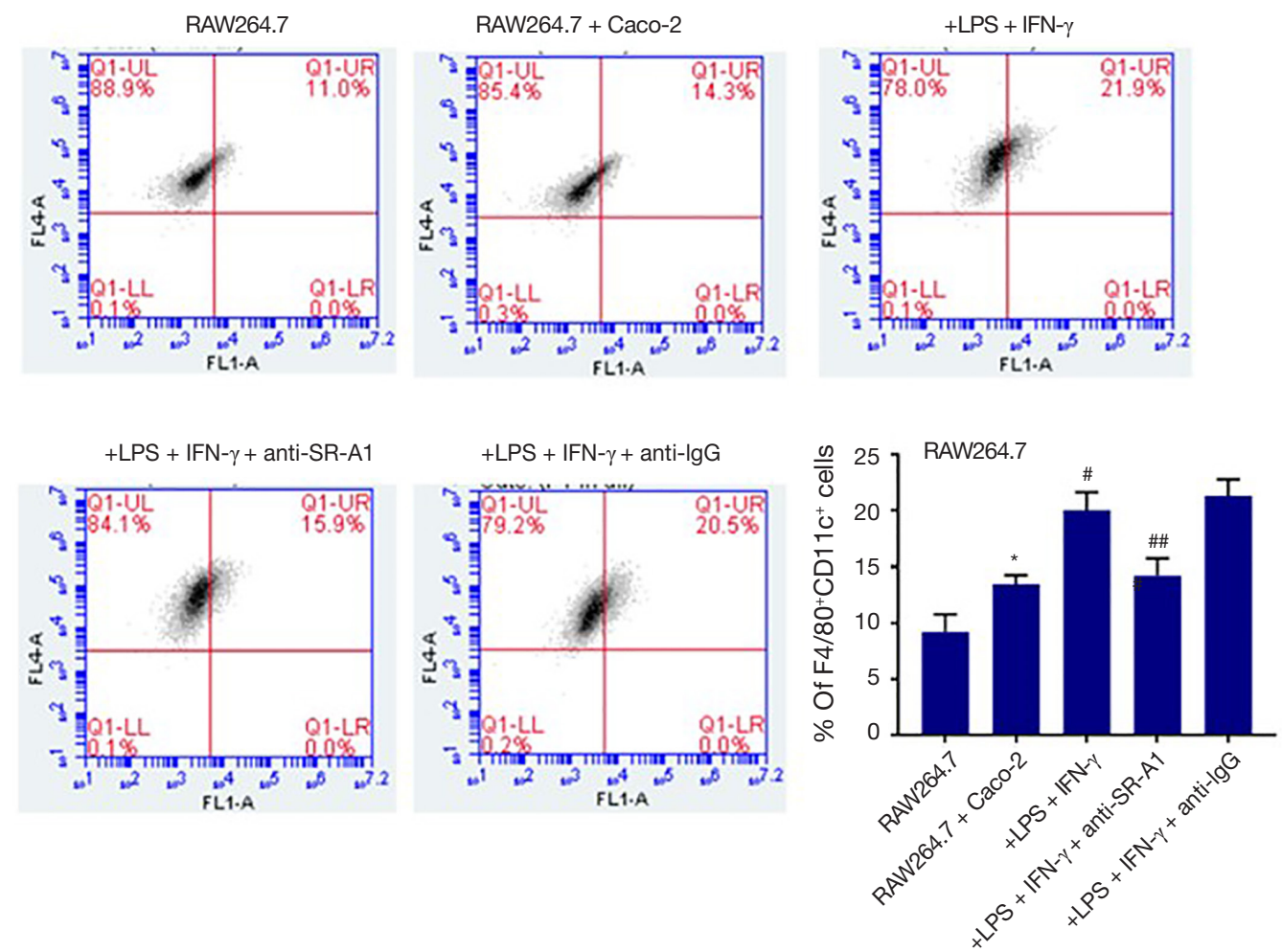

Figure 4 SR-A1 antibody pre-treatment inhibits M1 phenotype of RAW264.7 cells in the co-culture system. The F4/80 ${ }^{+} \mathrm{CD} 11 \mathrm{c}^{+}$ macrophages were detected by flow cytometry. Data were presented as mean \pm SEM $\left(^{*}, \mathrm{P}<0.05\right.$ vs. RAW264.7 cells group; ${ }^{\#}, \mathrm{P}<0.05$ vs. RAW264.7 cells + Caco-2 group; ${ }^{\#}, \mathrm{P}<0.05$ vs. LPS + IFN- $\gamma$ group). SR-A1, A1 scavenger receptor; SEM, standard error of the mean; LPS, lipopolysaccharide.

caspase-3 (17). In our co-culture system, colonic epithelial cells Caco-2 in different groups were stained with DAPI and photographed under a fluorescence microscope. As shown in Figure 6, untreated cells had homogeneous nuclei, but Caco-2 cells co-cultured with RAW264.7 cells with LPS and IFN- $\gamma$ treatment exhibited more condensed nuclei and apoptotic bodies after DAPI staining. SR-A1 antibody pre-treatment could attenuate the pro-apoptotic effect of macrophages on Caco- 2 cells, which was demonstrated by less condensed nuclei and less apoptotic bodies in cells.

In addition, the expression of cleaved caspase- 3 in Caco-2 cells was detected by Western blotting, and results indicated the cleaved caspase- 3 expression increased significantly in Caco- 2 cells after treatment with LPS and IFN- $\gamma$, which was also suppressed in the presence of SR-A1 antibody pre- treatment (Figure 7).

\section{Effects of SR-A1 antibody on tight junction protein expression in Caco-2 cells in the co-culture system}

Tight junction is an essential permeable intercellular barrier and plays a critical role in the intestinal epithelial barrier integrity, which can be disrupted by mucosal inflammation in the pathogenesis of IBD $(18,19)$. To determine the influence of SR-A1 antibody on tight junction protein in Caco-2 cells in the co-culture system, the expression of tight junction protein was detected in Caco-2 cells of coculture system. Our results indicated the protein expression of claudin-1 increased significantly after treatment with LPS and IFN- $\gamma$, which was markedly inhibited by the pre- 

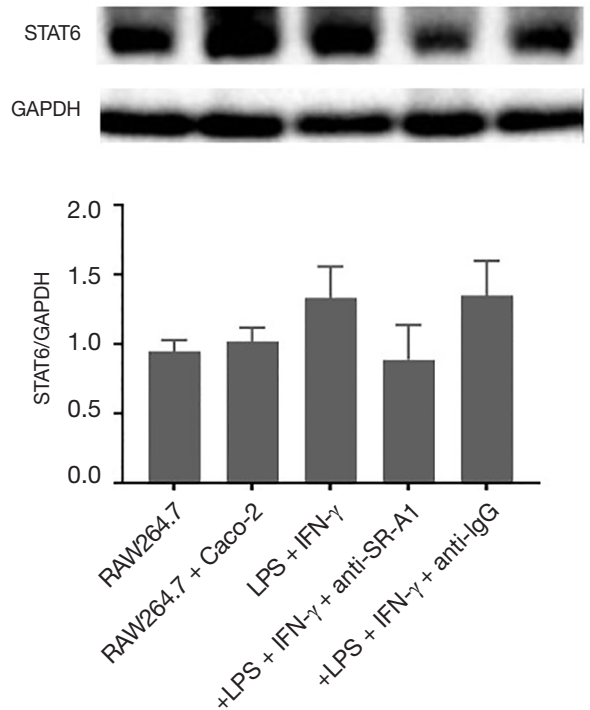

Figure 5 STAT6 expression of RAW264.7 cells in the co-culture system. Protein expression was detected by Western blotting. Data are presented as mean \pm SEM. SEM, standard error of the mean; SR-A1, A1 scavenger receptor; LPS, lipopolysaccharide. treatment with SR-A1 antibody (Figure 8).

\section{Discussion}

As the first-line defense in the lamina propria of mucosa, intestinal macrophages contribute to the integrity of intestinal barrier. On one hand, intestinal macrophages can eliminate invading pathogens though secreting cytokines and chemokines; on the other hand, intestinal macrophages are tolerant toward commensal microbiota though downregulating recognition receptors (20,21). Macrophages have different subtypes that can orchestrate or counteract inflammation, including pro-inflammatory M1 macrophages and anti-inflammatory M2 macrophages. Macrophages of different phenotypes have been investigated in many inflammation-associated diseases, including IBD. In CD patients, intestinal macrophages, especially a large number of $\mathrm{CD} 68^{+}$macrophages, usually migrate through the thickened mucosa to the submucosa. In UC patients, macrophages usually infiltrate the intestinal mucous layer
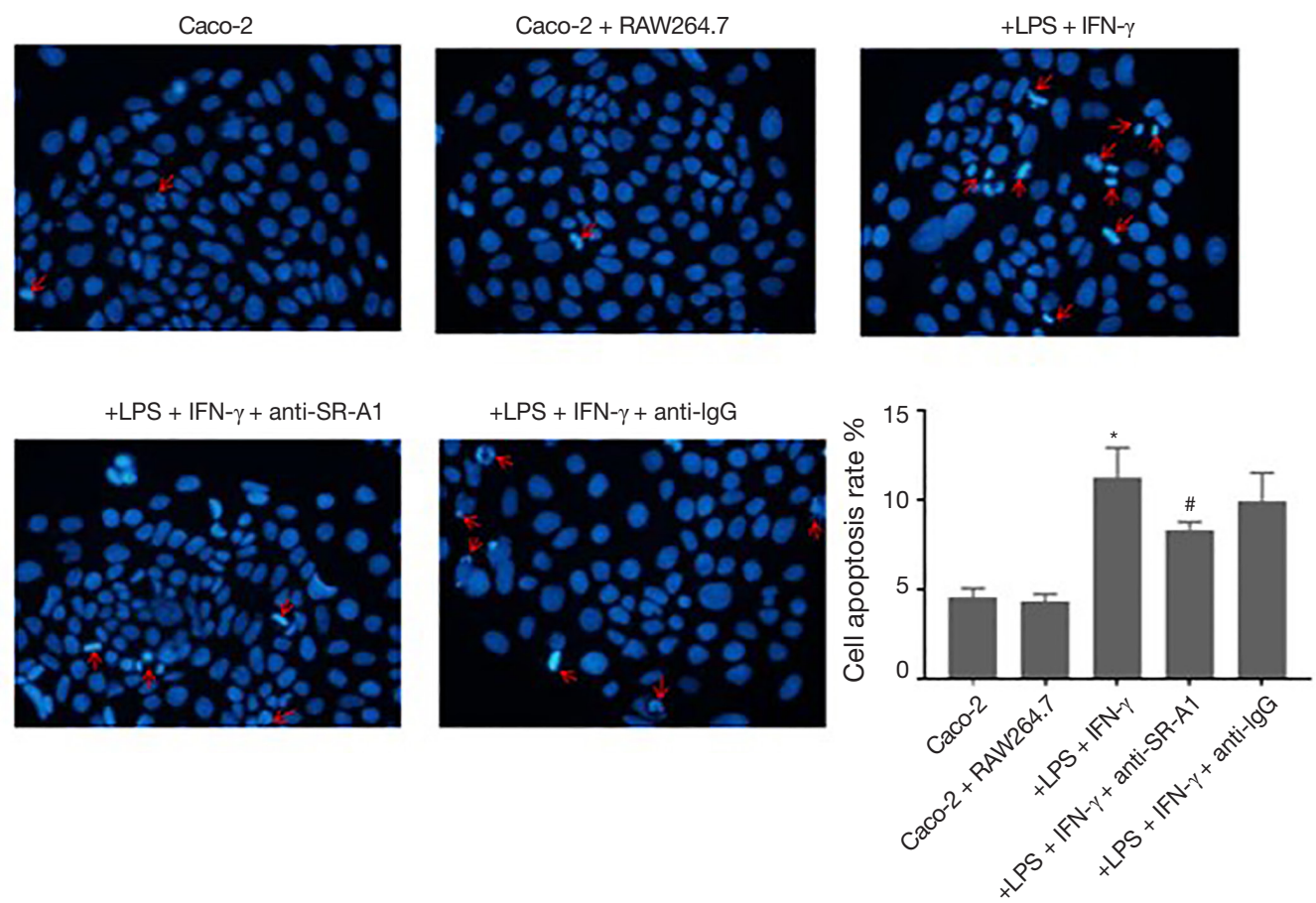

Figure 6 SR-A1 antibody pre-treatment attenuates the pro-apoptotic effect of RAW264.7 cells on Caco-2 cells in the co-culture system. Caco-2 in different groups were stained with DAPI and photographed under a fluorescence microscope $(\times 100)$. Red arrow: condensed nuclei and apoptotic bodies in Caco-2 cells. Data are presented as mean \pm SEM $\left(^{*}, \mathrm{P}<0.05\right.$ vs. RAW264.7 cells + Caco-2 group; ${ }^{\#}, \mathrm{P}<0.05$ vs. LPS + IFN- $\gamma$ group). SR-A1, A1 scavenger receptor; SEM, standard error of the mean; LPS, lipopolysaccharide. 
Cleaved caspase-3

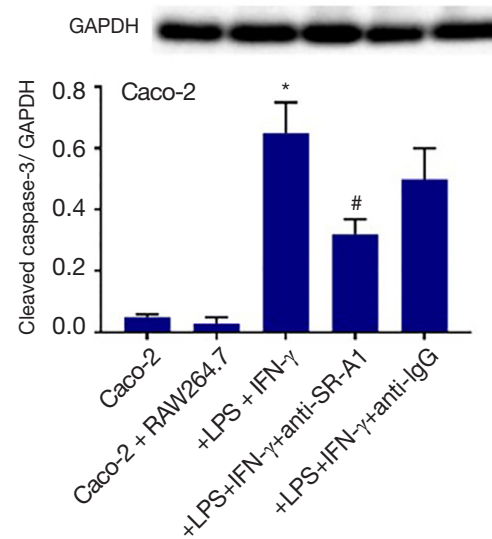

Figure 7 Expression of caspase- 3 in Caco-2 cells in the co-culture system. Protein expression was detected by Western blotting. Data are presented as mean \pm SEM. ${ }^{*}, \mathrm{P}<0.05$ vs. RAW264.7 cells + Caco-2 group; *, $\mathrm{P}<0.05$ vs. LPS $+\mathrm{IFN}-\gamma$ group). SEM, standard error of the mean; LPS, lipopolysaccharide; SR-A1, A1 scavenger receptor.

$(22,23)$. However, the exact mechanism underlying the role of human macrophages in the pathogenesis of IBD is still poorly understood.

In order to protect from the invasion of pathogens in the process of mucosal immune, macrophages secrete a variety of cytokines and exert strong anti-bactericidal effect via PRRs. SR-A1, as one of PRRs, is a type II membrane glycoprotein and can form homotrimers, involving in the progression of innate immunity, cell apoptosis and proliferation (24). SR-A1 is mainly expressed in macrophages, monocytes, dendritic cells, smooth muscle cells and endothelial cells, and can form complexes with other PRRS, exerting synergistic effects on the inflammatory response $(25,26)$. Early studies indicate SRA1 implicates in the pathogenesis of atherosclerosis, by mediating endocytotic effect of foam cells on low-density lipoprotein (27). Upon the cytomegalovirus exposure, SRA1, coordinating with Toll-like receptor 3/9, may induce the THP- 1 cells to express IL-12p35, IFN- $\beta$ and TNF, triggering pro-inflammatory response (28). In the present study, the mRNA expression of macrophage-related cytokines was detected in the presence of LPS in vitro, and results indicated pro-inflammatory cytokines, TNF and iNOS increased after LPS stimulation. They were down- regulated in the presence of SR-A1 antibody pre-treatment, indicating SR-A1 pathway plays a crucial role in the inflammatory response mediated by active macrophages. Furthermore, IL-10, which is one of factors associated with M2 macrophage polarization, can mediate mucosal tolerance by expanding antigen-specific FoxP $3^{+}$Treg in the intestinal mucosa (29). Our study showed SR-A1 antibody pre-treatment could up-regulate IL-10 expression in RAW264.7 cells, which suggests SR-A1 facilitates the inflammatory response not only by inducing the secretion of pro-inflammatory cytokines but also by suppressing the expression of anti-inflammatory cytokines.

The effect of macrophages on the bacterial clearance and endocytosis is associated with the expression of some inflammatory transcript factors, such as NF- $\mathrm{B}$, which can translocate into nucleus to promote the transcription of pro-inflammatory mediators (30,31). Nakamura et al. (32) reported that fucoidan could bind to SR-A1 to induce $\mathrm{NO}$ production in macrophages via $\mathrm{NF}-\kappa \mathrm{B}$ pathway. In an animal model of experimental brain injury, blockage of SR-A1 reduced the area of hippocampal neuronal damage, accompanied by the decreased NF- $\kappa \mathrm{B}$ activity (33). In our study, the expression of $\mathrm{NF}-\kappa \mathrm{B}$ p-p56 was detected by immunofluorescence staining, and results showed $\mathrm{NF}-\kappa \mathrm{B}$ p-p56 was mainly found in the nuclei, the NF- $\mathrm{B}$ p-p56 expression was up-regulated after LPS stimulation, which was markedly inhibited in the presence of SR-A1 antibody pre-treatment. These findings indicate the inflammatory process induced by macrophages via SR-A1 receptor is

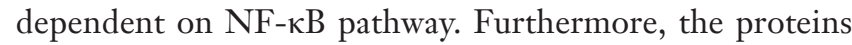
associated with macrophages proliferation were further detected, and results revealed the p-JNK expression, which was inhibited by SR-A1 antibody. Therefore, we hypothesize that SR-A1 may promote the proliferation of active macrophages via the JNK related pathway.

Studies have shown that the pathologic interactions between intestinal epithelial cells and macrophages may result in mucosal damage because of intestinal macrophages in close proximity to both the intestinal epithelium and luminal microbiota (34). Lissner et al. (18) reported M1 macrophages could reduce the transepithelial resistance as a marker for epithelial barrier integrity, involving in the deregulation of tight junction proteins and induction of epithelial cell apoptosis. Increasing studies have focused on the contribution of SRs to macrophage polarization. Accompanied by a decrease in M1 macrophages and an increase in M2 macrophages, SR-A1-deficient mice showed less infarct area in the brain injury model (35). In addition, 

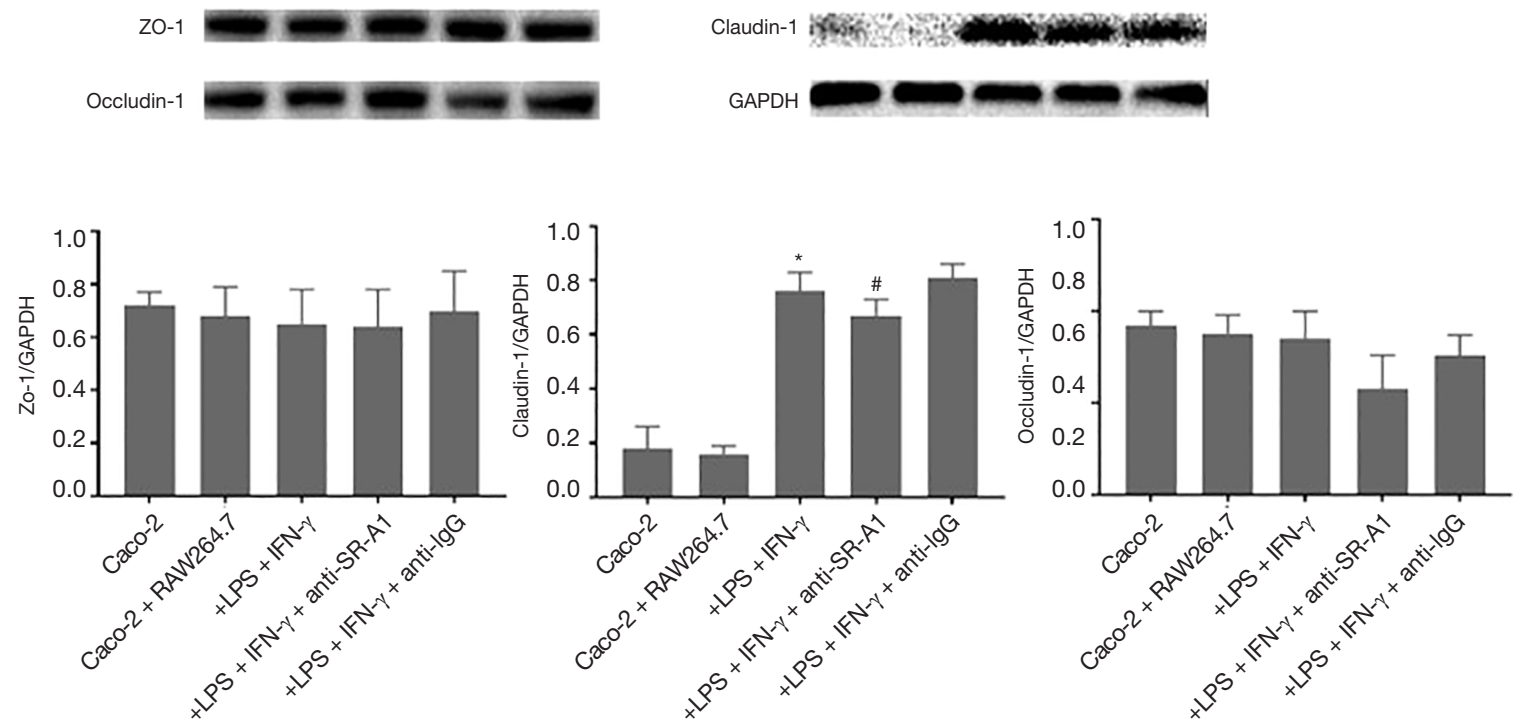

Figure 8 Expression of tight junction proteins in Caco-2 cells in the co-culture system. Protein expression was detected by Western blotting. Data are presented as mean \pm SEM. ( ${ }^{*}, \mathrm{P}<0.05$ vs. RAW264.7 cells + Caco-2 group; ${ }^{*}, \mathrm{P}<0.05$ vs. LPS + IFN- $\gamma$ group). SEM, standard error of the mean; LPS, lipopolysaccharide; SR-A1, A1 scavenger receptor.

M-BSA, a pan-class SR-A competitive inhibitor, was also found to suppress cytokine production in splenic M1-type macrophages (10). In the present study, RAW264.7 cells were co-cultured with Caco-2 cells, and the correlation between inflammatory response of macrophages and intestinal barrier integrity was further evaluated. The $\mathrm{F} 4 / 80^{+} \mathrm{CD} 11 \mathrm{c}^{+}$macrophages, which is a marker of $\mathrm{M} 1$ macrophage, were detected by flow cytometry. Results showed the percentage of $\mathrm{F} 4 / 80^{+} \mathrm{CD} 11 \mathrm{c}^{+}$macrophages increased after treatment with LPS and IFN- $\gamma$, which, however, was reversed by SR-A1 antibody pre-treatment, which suggests SR-A1 involves in M1 polarization associated with intestinal pro-inflammatory immune. There is evidence showing the induction of macrophage polarization is dependent on STAT6 pathway (36). However, there was no significant difference in STAT6 expression among groups in the present study.

Finally, the apoptosis rate of Caco-2 cells was determined, and the potential mechanism was further explored in this co-culture system. Our results showed pretreatment with SR-A1 antibody could attenuate the proapoptotic effect of macrophages on Caco-2 cells in the presence of LPS and IFN- $\gamma$, and the expression of cleaved caspase-3 reduced. It is proposed that SR-A1 is necessary to activate M1-induced apoptosis in a caspase-3 dependent manner. Claudin-1 functions as a major constituent of the tight junction complexes and regulate the permeability of epithelia, which plays an essential role in the formation of impermeable barriers $(37,38)$. Our results showed claudin-1 expression in Caco-2 cells increased in the presence of LPS and IFN- $\gamma$, which was inhibited by the SR-A1 antibody pretreatment, in accordance with the redistribution of tight junction proteins to the cytoplasm or basal parts of the epithelial cells after incubation with M1 macrophages (39).

In summary, SR-A1 antibody pre-treatment upregulates anti-inflammatory cytokines and downregulates inflammatory cytokines secreted by LPS-treated macrophages, which is associated with NF- $\mathrm{kB}$ pathway. SRA1 antibody pre-treatment also inhibits M1 phenotype of macrophages, and attenuates the pro-apoptotic effect of macrophages on colonic epithelial cells and the disruption of intestinal barrier integrity. Our study indicates SR-A1 can impair intestinal barrier function via inducing the M1 phenotype of macrophages in the pathogenesis of IBD.

\section{Acknowledgments}

Funding: This work was supported by the National Natural Science Foundation of China (No.81770558) and Xiamen Joint Projects for Major Disease (3502z20149031) 
to Jianlin Ren.

\section{Footnote}

Conflict of Interest: The authors have no conflicts of interest to declare.

Ethical Statement: The authors are accountable for all aspects of the work in ensuring that questions related to the accuracy or integrity of any part of the work are appropriately investigated and resolved.

\section{References}

1. Ramos GP, Papadakis KA. Mechanisms of disease: inflammatory bowel diseases. Mayo Clin Proc 2019;94:155-65.

2. Zuo T, Ng SC. The gut microbiota in the pathogenesis and therapeutics of inflammatory bowel disease. Front Microbiol 2018;9:2247.

3. Iida T, Yokoyama Y, Wagatsuma K, et al. Impact of autophagy of innate immune cells on inflammatory bowel disease. Cells 2018. doi: 10.3390/cells8010007.

4. Bain CC, Schridde A. Origin, differentiation, and function of intestinal macrophages. Front Immunol 2018;9:2733.

5. Xuan W, Qu Q, Zheng B, et al. The chemotaxis of M1 and M2 macrophages is regulated by different chemokines. J Leukoc Biol 2015;97:61-9.

6. Ingersoll MA, Spanbroek R, Lottaz C, et al. Comparison of gene expression profiles between human and mouse monocyte subsets. Blood 2010;115:e10-9.

7. Kunjathoor VV, Febbraio M, Podrez EA, et al. Scavenger receptors class A-I/II and CD36 are the principal receptors responsible for the uptake of modified low density lipoprotein leading to lipid loading in macrophages. J Biol Chem 2002;277:49982-8.

8. Ren D, Wang X, Ha T, et al. SR-A deficiency reduces myocardial ischemia/reperfusion injury; involvement of increased microRNA-125b expression in macrophages. Biochim Biophys Acta 2013;1832:336-46.

9. Sapkota M, DeVasure JM, Kharbanda KK, et al. Malondialdehyde-acetaldehyde (MAA) adducted surfactant protein induced lung inflammation is mediated through scavenger receptor a (SR-A1). Respir Res 2017;18:36.

10. Komai K, Shichita T, Ito M, et al. Role of scavenger receptors as damage-associated molecular pattern receptors in Toll-like receptor activation. Int Immunol 2017;29:59-70.
11. Kim KM, Kim YS, Lim JY, et al. Intestinal antiinflammatory activity of Sasa quelpaertensis leaf extract by suppressing lipopolysaccharide-stimulated inflammatory mediators in intestinal epithelial Caco-2 cells co-cultured with RAW 264.7 macrophage cells. Nutr Res Pract 2015;9:3-10.

12. De Schepper S, Verheijden S, Aguilera-Lizarraga J, et al. Self-maintaining gut macrophages are essential for intestinal homeostasis. Cell 2018;175:400-15.e13.

13. MacDonald TT, Monteleone I, Fantini MC, et al. Regulation of homeostasis and inflammation in the intestine. Gastroenterology 2011;140:1768-75.

14. Li X, Luo H, Ye Y, et al. betaglucan, a dectin1 ligand, promotes macrophage M1 polarization via NFkappaB/ autophagy pathway. Int J Oncol 2019;54:271-82.

15. Kühl AA, Erben U, Kredel LI, et al. Diversity of intestinal macrophages in inflammatory bowel diseases. Front Immunol 2015;6:613.

16. Yan D, Wang HW, Bowman RL, et al. STAT3 and STAT6 signaling pathways synergize to promote cathepsin secretion from macrophages via IRE1alpha activation. Cell Rep 2016;16:2914-27.

17. Manning-Tobin JJ, Moore KJ, Seimon TA, et al. Loss of SR-A and CD36 activity reduces atherosclerotic lesion complexity without abrogating foam cell formation in hyperlipidemic mice. Arterioscler Thromb Vasc Biol 2009;29:19-26.

18. Lissner D, Schumann M, Batra A, et al. Monocyte and M1 Macrophage-induced barrier defect contributes to chronic intestinal inflammation in IBD. Inflamm Bowel Dis 2015;21:1297-305.

19. Lu JT, Xu AT, Shen J, et al. Crosstalk between intestinal epithelial cell and adaptive immune cell in intestinal mucosal immunity. J Gastroenterol Hepatol 2017;32:975-80.

20. Mann ER, Li X. Intestinal antigen-presenting cells in mucosal immune homeostasis: crosstalk between dendritic cells, macrophages and B-cells. World J Gastroenterol 2014;20:9653-64.

21. Noel G, Baetz NW, Staab JF, et al. A primary human macrophage-enteroid co-culture model to investigate mucosal gut physiology and host-pathogen interactions. Sci Rep 2017;7:45270.

22. Wehner S, Engel DR. Resident macrophages in the healthy and inflamed intestinal muscularis externa. Pflugers Arch 2017;469:541-52.

23. Grainger JR, Konkel JE, Zangerle-Murray T, et al. Macrophages in gastrointestinal homeostasis and 
inflammation. Pflugers Arch 2017;469:527-39.

24. Prabhudas M, Bowdish D, Drickamer K, et al. Standardizing scavenger receptor nomenclature. J Immunol 2014;192:1997-2006.

25. Ben J, Zhu X, Zhang H, et al. Class A1 scavenger receptors in cardiovascular diseases. Br J Pharmacol 2015;172:5523-30.

26. Kim WS, Ordija CM, Freeman MW. Activation of signaling pathways by putative scavenger receptor class A (SR-A) ligands requires CD14 but not SR-A. Biochem Biophys Res Commun 2003;310:542-9.

27. Watanabe K, Nakazato Y, Saiki R, et al. Acroleinconjugated low-density lipoprotein induces macrophage foam cell formation. Atherosclerosis 2013;227:51-7.

28. Yew KH, Carsten B, Harrison C. Scavenger receptor A1 is required for sensing HCMV by endosomal TLR-3/-9 in monocytic THP-1 cells. Mol Immunol 2010;47:883-93.

29. Shapouri-Moghaddam A, Mohammadian S, Vazini H, et al. Macrophage plasticity, polarization, and function in health and disease. J Cell Physiol 2018;233:6425-40.

30. Zhao Y, Ma G, Yang X. HDAC5 promotes mycoplasma pneumoniae-induced inflammation in macrophages through NF-kappaB activation. Life Sci 2019;221:13-9.

31. Wang H, Zhang L, Xu S, et al. Surface-layer protein from lactobacillus acidophilus NCFM inhibits lipopolysaccharide-induced inflammation through MAPK and NF-kappaB signaling pathways in RAW264.7 cells. J Agric Food Chem 2018;66:7655-62.

Cite this article as: Xie C, Fan Y, Huang Y, Wu S, Xu H, Liu L, Hu Y, Huang Q, Shi H, Wang L, Xu H, Su J, Ren J. Class A1 scavenger receptors mediated macrophages in impaired intestinal barrier of inflammatory bowel disease. Ann Transl Med 2020;8(4):106. doi: 10.21037/atm.2019.12.107
32. Nakamura T, Suzuki H, Wada Y, et al. Fucoidan induces nitric oxide production via p38 mitogen-activated protein kinase and NF-kappaB-dependent signaling pathways through macrophage scavenger receptors. Biochem Biophys Res Commun 2006;343:286-94.

33. Lu C, Hua F, Liu L, et al. Scavenger receptor class-A has a central role in cerebral ischemia-reperfusion injury. J Cereb Blood Flow Metab 2010;30:1972-81.

34. Barman S, Kayama H, Okuzaki D, et al. Identification of a human intestinal myeloid cell subset that regulates gut homeostasis. Int Immunol 2016;28:533-45.

35. Xu Y, Qian L, Zong G, et al. Class A scavenger receptor promotes cerebral ischemic injury by pivoting microglia/ macrophage polarization. Neuroscience 2012;218:35-48.

36. Ding N, Wang Y, Dou C, et al. Physalin D regulates macrophage M1/M2 polarization via the STAT1/6 pathway. J Cell Physiol 2019;234:8788-96.

37. Zorn-Kruppa M, Vidal-Y-Sy S, Houdek P, et al. Tight junction barriers in human hair follicles - role of claudin-1. Sci Rep 2018;8:12800.

38. Hu CA, Hou Y, Yi D, et al. Autophagy and tight junction proteins in the intestine and intestinal diseases. Anim Nutr 2015;1:123-7.

39. Stamatovic SM, Johnson AM, Sladojevic N, et al. Endocytosis of tight junction proteins and the regulation of degradation and recycling. Ann N Y Acad Sci 2017;1397:54-65. 